\title{
INCIDÊNCIA DE ENDOPARASITAS E ECTOPARASITAS EM EQUINOS DO MUNICÍPIO DE CURITIBA - PR
}

\author{
Incidence of endoparasites and ectoparasites in equines from \\ the city of Curitiba - Parana
}

\footnotetext{
Daiane Xavier Rego ${ }^{[a]}$, Bruno de Resende Pedroso Schmeil ${ }^{[b]}$, Juliana Weber Schiller ${ }^{[c]}$, Mariana Müeller da Silva ${ }^{[\mathrm{d}]}$, Cassiana Garcez $\operatorname{Ramos}^{[\mathrm{e}]}$, Pedro Vicente Michelotto Júnior ${ }^{[\mathrm{f}]}$

[a] Discente de graduação do Curso de Medicina Veterinária da Pontifícia Universidade Católica do Paraná (PUCPR), Curitiba, PR - Brasil, e-mail: dai_vet@hotmail.com

${ }^{[b]}$ Discente de graduação do Curso de Medicina Veterinária da Pontifícia Universidade Católica do Paraná (PUCPR), Curitiba, PR -Brasil, e-mail: b_schmeil@yahoo.com.br

${ }^{[c]}$ Discente de graduação do Curso de Medicina Veterinária da Pontifícia Universidade Católica do Paraná (PUCPR), Curitiba, PR -Brasil,e-mail: juschiller@yahoo.com.br

[d] Médica Veterinária residente da Unidade Hospitalar para Equinos da Pontifícia Universidade Católica do Paraná (PUCPR), Curitiba, PR - Brasil, e-mail: mari.mm.s@gmail.com

${ }^{[e]}$ Professora de Anestesiologia do Curso de Medicina Veterinária da Pontifícia Universidade Católica do Paraná (PUCPR), Curitiba, PR - Brasil, e-mail: cassyj@terra.com.br

${ }^{[\mathrm{f}]}$ Professor de Doenças Parasitárias de Animais de Fazenda e Equinos e de Semiologia, Clínica Médica e Cirúrgica de Equinos, Curso de Medicina Veterinária da Pontifícia Universidade Católica do Paraná (PUCPR), Curitiba, PR - Brasil, e-mail: michelottojunior@yahoo.com.br
}

\begin{abstract}
Resumo
O objetivo do presente estudo foi o de avaliar a incidência de endoparasitas e ectoparasitas na população de equinos no município de Curitiba, Paraná, com a finalidade, também, de se obter um panorama atual a respeito das parasitoses de equinos nesta região. Foram avaliados 166 equinos, sendo 53 fêmeas e 113 machos, em cinco diferentes propriedades, utilizados para trabalho e esporte, dentro do município de Curitiba, incluindo animais de diferentes raças e idades. Estabeleceu-se uma ficha clínica para as anotações referentes ao histórico e ao exame clínico de cada animal. Foi realizada a colheita de fezes para a elaboração de exame coproparasitológico, pelas técnicas de OPG e Flutuação e foi procedida a colheita de sangue, para a avaliação hematológica. Dentre os animais avaliados, 55 deles apresentaramse positivos pela técnica de OPG e, destes, 23 animais tiveram OPG acima de 300 ovos/g de fezes. Na avaliação pela técnica de flutuação, um total de 61 animais apresentaram-se positivos, e destes, 43 animais resultaram em flutuação acima de $3+$. Nem todos esses equinos parasitados foram identificados em ambas as técnicas empregadas, sendo que, dentre eles, cinco apresentaram-se positivos apenas na avaliação por OPG enquanto que dez apresentaram-se positivos unicamente quando a avaliação foi
\end{abstract}


pela técnica de flutuação. Foram encontrados quatro tipos de parasitas nas avaliações, sendo o Strongilus sp. (58 animais), o Trichostrongylus (16 animais), o Oxyuris (25 animais) e o Parascaris (8 animais). O grupo de cavalos parasitados apresentou hematócrito significativamente inferior aos cavalos negativos nas avaliações coproparasitológicas $(\mathrm{p}<0,01)$. Igualmente, apresentaram os valores de proteína plasmática $(\mathrm{p}<0,0001)$ e de fibrinogênio $(\mathrm{p}<0,05)$ significativamente maiores em comparação aos cavalos não parasitados. Dois animais apresentaram-se com Babesiose, enquanto que ectoparasitas não foram encontrados em nenhum dos animais avaliados. No presente estudo, pode-se constatar que as parasitoses ainda continuam presentes na população de equinos, no município de Curitiba, resultando em alterações hematológicas importantes. Da mesma forma, como se tem recomendado, faz-se importante realizar os exames coproparasitológios rotineiramente e utilizando as técnicas de OPG e flutuação conjuntamente, para que não haja engano no diagnóstico.

Palavras-chave: Equino. Parasitas. Endoparasitas. Ectoparasitas. Babesia.

\begin{abstract}
The present study aimed to evaluate the incidence of endo and ectoparasites in the equine population from the city of Curitiba, Paraná State. Sport and pleasure horses from five different properties were evaluated, including distinct breeds and ages. A clinical report file was established and the basic information of each evaluated horse was jotted down. The faeces were evaluated by flotation and EPG techniques and blood was collected for hematologic evaluation. A total of 166 horses were examined, being 53 females and 113 males. Between them, 55 horses were positive when examined by the EPG technique, being that 23 had more than 300 eggs/gram of faeces. Performing the flotation technique, 61 horses were positive, and 43 evidenced at least 3+. Not all from these horses positive for parasites were identified on both techniques, being that five of them were positive only by the EPG procedure as 10 were found positive only in the flotation technique. Four different parasites were identificated, were Strongilus sp., Trichostrongylus, Oxyuris and Parascaris. From the total 58 horses affected by Strongylus, 16 horses by Trichostrongylus, 25 horses by Oxyuris and 8 horses by Parascaris. Babesiosis was encountered in the blood smear of two of the evaluated horses. Ectoparasites were not observed. In conclusion, the present study evidenced that parasitosis are still prevalent in the equine population from the city of Curitiba, as it has being reported in other countries. Finally, it was evident the necessity of routinely performing faeces examination, being necessary the utilization of both the EPG and flotation techniques, in order to avoid diagnostic mistakes.
\end{abstract}

Keywords: Equine. Parasites. Endoparasites. Ectoparasites. Babesia.

\title{
INTRODUÇÃO
}

Os equinos continuam sendo bastante acometidos por parasitas, os quais causam diversos sintomas e doenças. Os endoparasitas, encontrados nos diversos sistemas pelos diferentes ciclos migratórios, resultam em variadas enfermidades, tais como gastrites, enterites, nefrites, hepatite e broncopneumonia, entre outras (BORCHERT, 1975; BOWMAN, 1995; FORTES, 2004; GRAY, 1995; URQUHART, 1998).

Os parasitas gastrointestinais, geralmente apresentam-se de forma subclínica, acarretando perdas econômicas significativas em animais utilizados para trabalho, esporte e reprodução (KNOTTENBELT, 1998; RIET-CORREIA et al., 2001; SMITH, 1994). Somando aos seus efeitos 
deletérios, muitos têm demonstrado o desenvolvimento de resistência aos programas e princípios antiparasitários disponíveis (CRAIG et al., 2007; LINDGREN et al., 2008; SAMSON-HIMMELSTJERNA et al., 2007; SLOCOMBE et al., 2007).

Nos grupos de potros jovens, o Parascaris equorum tem se apresentado de forma frequente pela sua natureza cosmopolita (LINDGREN et al., 2008). Sua infecção ocorre a partir da ingestão de ovos durante o pastejo, causando sinais clínicos respiratórios, perda do apetite, fraqueza, prejuízos ao crescimento, enterite, e ocasionalmente obstrução e peritonite (BOYLE; HOUSTON, 2006).

O pastejo intensivo também tem resultado na resistência de Strongylus aos antiparasitários, incluindo as lactonas macrocíclicas (KAPLAN, 2004).

Assim, devido a diversos relatos do aparecimento de formas parasitárias resistentes em diversos países, objetivou-se, no presente estudo, obter informações sobre a prevalência dos endoparasitas, observando-se também os ectoparasitas em equinos da região de Curitiba, Paraná.

\section{MATERIAIS E MÉTODO}

Foram avaliados equinos de diferentes idades, machos e fêmeas, provenientes de cinco diferentes estabelecimentos do município de Curitiba.

A avaliação foi procedida através de exame físico completo (frequência cardíaca, frequência respiratória, coloração de mucosas, tempo de preenchimento capilar, qualidade de pulso arterial e palpação de linfonodos) (FEITOSA, 2004).

Após o exame físico, de cada cavalo foi colhido sangue em frasco com EDTA (vacutainer ${ }^{\circledR}$ ), por venopunção jugular, para a avaliação hematológica, procedida no laboratório de análises clínicas da unidade hospitalar para equinos do Hospital Veterinário da PUCPR.

As avaliações coproparasitológicas foram realizadas com amostra de fezes colhidas também durante a avaliação inicial, por colheita da ampola retal. Realizou-se avaliação por meio das técnicas de McMaster (GORDON; WHITLOCK, 1939) que permite a identificação e contagem de OPG em fezes de equinos e flutuação, no período máximo de 6 horas após a colheita.

A análise estatística foi realizada por meio do teste de Mann-Whitney, sendo que foi considerado significativo um valor de $\mathrm{p}<0,05$. Os valores estão expressos como média \pm erro padrão da média.

\section{RESULTADOS}

Foram avaliados 166 equinos de cinco diferentes propriedades, utilizados para trabalho e esporte, incluindo animais de diferentes raças e idades, sendo 53 (31,9\%) fêmeas e $113(68,1 \%)$ machos.

Dentre eles, $55(33,1 \%)$ animais apresentaram-se positivos pela técnica de OPG, e destes, $23(41,8 \%)$ animais tiveram OPG acima de 300 ovos/g de fezes. Na avaliação pela técnica de flutuação, um total de $61(36,8 \%)$ animais apresentou-se positivo, e destes, $43(70,5 \%)$ animais resultaram em flutuação acima de $3+$. Somente $5(3 \%)$ equinos apresentaram-se positivos apenas na avaliação por OPG enquanto que $10(6 \%)$ animais apresentaram-se positivos somente na flutuação. Foram encontrados quatro tipos de parasitas nas avaliações, sendo Strongylus sp., Trichostrongylus, Oxyuris e Parascaris.

Dentre os animais avaliados, $58(35 \%)$ deles apresentaram-se com Strongylus, $16(9,6 \%)$ equinos evidenciaram Trichostrongylus, $25(15,1 \%)$ deles Oxyuris e $8(4,8 \%)$ animais tiveram Parascaris.

O grupo de cavalos parasitados, identificados por uma ou outra técnica de avaliação, apresentou valores de hematócrito significativamente menores em comparação com os cavalos não parasitados $(38,4 \pm 0,58 \%$ vs. $40,5 \pm 0,50 \%)(\mathrm{p}<0,01)$ (Figura 1). 


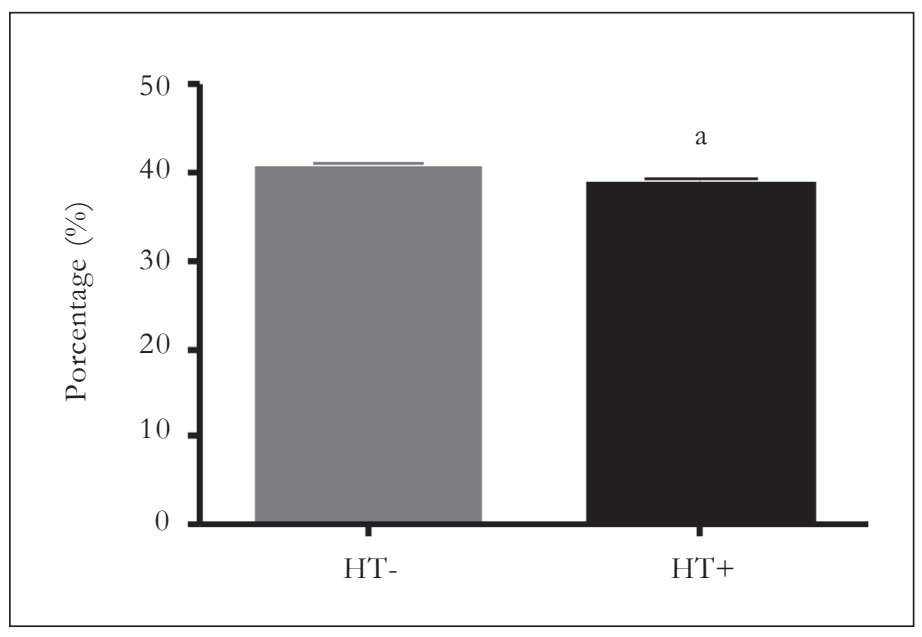

FIGURA 1 - Valores de hematócrito expressos em porcentagem, para os grupos de cavalos não parasitados (HT-) e parasitados (HT+). ${ }^{a} \mathrm{p}<0,01$ vs. HT-

Também, os animais parasitados apresentaram valores significativamente maiores de proteína plasmática $(9,5 \pm 0,17 \mathrm{~g} / \mathrm{dl}$ vs. $8,7 \pm 0,10 \mathrm{~g} / \mathrm{dl})(\mathrm{p}<0,0001)$ (Figura 2) e de fibrinogênio (425 $\pm 48,0 \mathrm{mg} / \mathrm{dl}$ vs. $271 \pm 16,4 \mathrm{mg} / \mathrm{dl})(\mathrm{p}<0,05)$ (Figura 3), em comparação com os cavalos não parasitados.

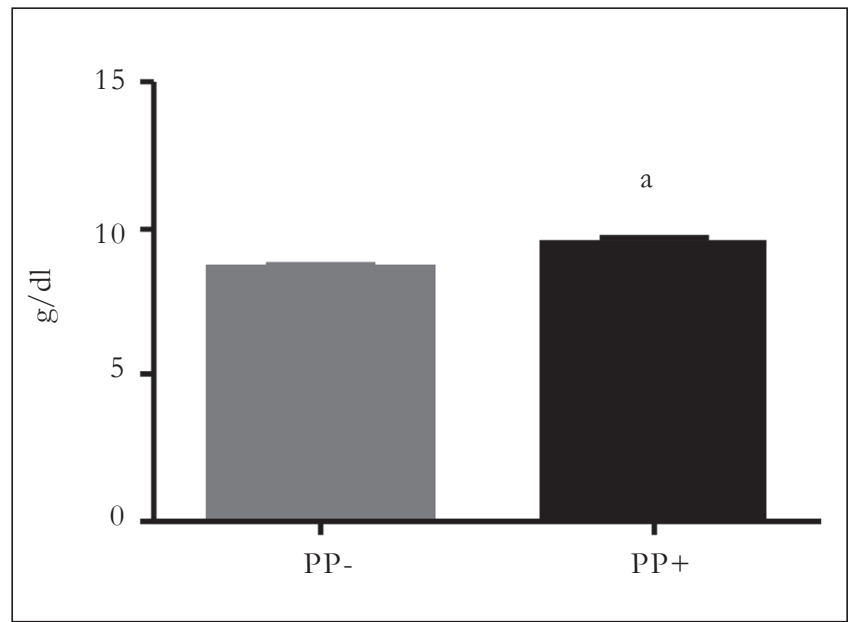

FIGURA 2 - Valores de proteína plasmática expressos em $\mathrm{g} / \mathrm{dl}$, para os grupos de cavalos não parasitados (PP-) e parasitados (PP+). ${ }^{a} \mathrm{P}$ $<0,0001$ vs. PP-

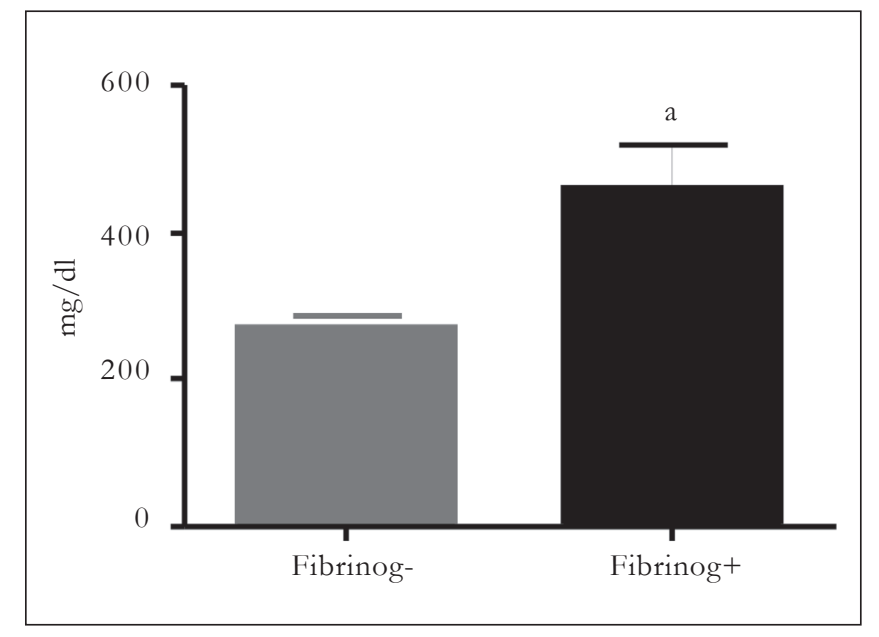

FIGURA 3 - Valores de fibrinogênio expressos em mg/ dl, para os grupos de cavalos não parasitados (Fibrinog -) e parasitados (Fibrinog + ). ${ }^{a} \mathrm{p}$ $<0,05$ vs. Fibrinog -

Dois $(1,2 \%)$ animais apresentaram-se com Babesiose, enquanto que ectoparasitas não foram encontrados em nenhum dos animais avaliados (Figura 1).

\section{DISCUSSÃO E CONCLUSÕES}

As técnicas empregadas para a avaliação coproparasitológica, no presente estudo, são as usualmente empregadas nos estudos de detecção parasitária, apresentando-se confiáveis (CRAIG et al., 
2007; SLOCOMBE; GANNES; LAKE, 2007), apesar de haver técnicas de maior precisão, mas ainda não disponíveis a todos os clínicos, tais como PCR (HODGKINSON, 2006; NIELSEN et al., 2008) e ELISA (MATTHEWWS et al., 2004).

A detecção de endoparasitas pareceu efetiva com as técnicas utilizadas, podendo-se identificar diferentes espécies parasitárias apesar de não ter-se associada à cultura de larvas, o que certamente teria assegurada a identificação das espécies. Ademais, pode-se comprovar a importância da realização de mais de uma técnica de forma conjunta, sendo que outros autores demonstraram a detecção e o controle das infestações parasitárias utilizando as técnicas empregadas no presente estudo (LIND et al., 2007; NIELSEN et al., 2008; PEREGRINE et al., 2005).

Contudo, para algumas espécies de parasitas, tais como a Anoplocephala perfoliata, as técnicas coproparasitológicas empregadas apresentam limitação na sua detecção, demonstrada quando do emprego de métodos mais apurados como o PCR (DROGEMULLER et al., 2004) e o ELISA (KANIA; REINEMEYER, 2005). Particularmente a $A$. perfoliata foi encontrada em $85 \%$ dos animais estudados no Vale do Paraíba, estado de São Paulo, mas com a detecção realizada em exame de necropsia (PEREIRA; VIANNA, 2006). Desta forma, possivelmente a detecção deste parasita tenha ficado subestimada no presente estudo.

Ainda no estudo de Pereira e Vianna (2006), foram encontrados nematódeos em 100\% dos animais estudados, havendo sido importante a incidência para cada uma das espécies, como o Cyathostomum (100\%), Oxyuris equi (90\%), Strongylus vulgaris (70\%), Strongylus edentatus (45\%), Strongylus equinus (15\%), Triodontophorus (60\%), Gyalocephalus capitatus (50\%), Oesophagodontus robustus (15\%), Craterostomum acuticaudatum (15\%), Parascaris equorum (5\%), Probstimayria vivipara (15\%), Habronema muscae $(15 \%)$ e Trichostrongylus axei (15\%).

Ainda assim, a prevalência de quatro espécies parasitárias foi importante em nosso estudo, visto que os animais avaliados eram cavalos sob bom manejo, incluindo animais atletas de alto nível. Possivelmente esse fato se deva à resistência a alguns antiparasitários que vêm sendo utilizados há bastante tempo, como é demonstrado para a ivermectina (SLOCOMBE et al., 2007) e/ou ao não controle da eficácia do programa antiparasitário estabelecido para a propriedade (LINDGREN et al., 2008; SAMSON-HIMMELSTJERNA et al., 2007; SLOCOMBE et al., 2007).

Essas espécies parasitárias, mesmo em infecções moderadas, resultam em fraqueza e inflamação intestinal (GRAY, 1995; LINDGREN et al., 2008). Os efeitos inflamatórios e espoliativos dos parasitas, no organismo dos cavalos, podem ser evidenciados nas avaliações hematológicas.

Com relação aos ectoparasitas, apesar de serem prevalentes em nosso país, incluindo os estados da região Sul (EVANS; MARTINS; GUGLIELMONE, 2000), não foram observados no presente estudo, possivelmente por se tratar de cavalos estabulados e sob manejo intensivo. Contudo, os hemoparasitas, representados pelo gênero Babesia, puderam ser encontrados somente em dois dos animais avaliados. Da mesma forma, a prevalência demonstrada foi abaixo dos relatos em nosso país, certamente pela técnica de detecção empregada, pela observação direta em esfregaço sanguíneo, a qual não apresenta a mesma acurácia em relação aos métodos imunológicos de detecção como o PCR (CRIADO et al., 2006), o ELISA (HUANG et al., 2006a) e a imunocromatografia (HUANG et al., 2006b), que resultaram na incidência de 40\% de babesiose nos equinos em nosso país (XUAN et al., 2001).

Assim, no presente estudo pode-se constatar que as parasitoses continuam presentes na população de equinos, no município de Curitiba, mesmo nas propriedades com bom manejo, corroborando com os relatos em diversos países. Da mesma forma, pode-se comprovar a utilidade das técnicas empregadas de detecção e também a importância da realização conjunta das técnicas de OPG e flutuação, para que não ocorra equívoco no diagnóstico.

\section{REFERÊNCIAS}

BORCHERT, A. Parasitología veterinaria. Zaragosa: Acribia, 1975.

BOWMAN, D. D. Georgis' parasitology for veterinarians. 6th ed. Philadelphia : W.B. Saunders, c1995. 
BOYLE, A. G.; HOUSTON, R. Parasitic pneumonitis and treatment in horses. Clinical Techniques in Small Animal Practice, New York, v. 5, n. 3, p. 225-232, 2006.

CRAIG, T. M. et al. Evidence of ivermectin resistance by Parascaris equorum on a Texas horse farm. Journal of Equine Veterinary Science, Wildomar, v. 27, n. 2, p. 67-71, 2007.

CRIADO, A. et al. New data on epizootiology and genetics of piroplasms based on sequences of small ribosomal subunit and cytochrome $b$ genes. Veterinary Parasitology, Amsterdam, v. 142, n. 3/4, p. 238-247, 2006.

DROGEMULLER, M. et al. Amplification of ribosomal DNA of Anoplocephalidae: Anoplocephala perfoliata diagnosis by PCR as a possible alternative to coprological methods. Veterinary Parasitology, Amsterdam, v. 124, n. 3/4, p. 205-215, 2004.

EVANS, D. E.; MARTINS, J. R.; GUGLIELMONE, A. A. A review of the ticks (Acari, Ixodida) of Brazil, their hosts and geographical distribution - 1. the State of Rio Grande do Sul, Southern Brazil. Memórias do Instituto Oswaldo Cruz, Rio de Janeiro, v. 95, n. 4, p. 453-470, 2000.

FEITOSA, F. L. F. Semiologia veterinária: a arte do diagnóstico. São Paulo: Roca, 2004.

FORTES, E. Parasitologia veterinária. 4. ed. São Paulo: Ícone, 2004.

GORDON, H. M.; WHITLOCK, H. U. A. New technique for counting nematode eggs in sheep feces. Journal Council Scientific Industry Research, Australia, v. 12, p. 50-52, 1939.

GRAY, P. Parasites and skin diseases. London: J. A. Allen, 1995.

HODGKINSON, J. E. Molecular diagnosis ande quine parasitology. Veterinary Parasitology, Amsterdam, v. 136, n. 2, p. 109-116, 2006.

HUANG, X. et al. Evaluation of ezyme-linked immunosorbent assays with recombinant antigens for the serodiagnosis of equine Babesia infections. Veterinary Parasitology, Amsterdam, v. 140, n. 1-2, p. 158-161, 2006a.

. Immunochromatographic test for simultaneous serodiagnosis of Babesia caballi and B. equi infections in horses. Clinical and Vaccine Immunology, Washington, v. 13, n. 5, p. 553-555, $2006 \mathrm{~b}$.

KANIA, S. A.; REINEMEYER, C. R. Anoplocephala perfoliata coproantigen detection: a preliminary study. Veterinary Parasitology, Amsterdam, v. 127, n. 2, p. 115-119, 2005.

KAPALN, R. Drug resistance in nematodes of veterinary importance: a status report. Trends Parasitology, Boston, v. 20, n. 10, p. 477-481, 2004.

KNOTTENBELT, D. C.; PASCOE, R. R. Afecções e distúrbios do cavalo. São Paulo: Manole, 1998.

LIND, E. O. et al. A field study on the effect of some anthelmintics on cyathostomins of horses in sweden. Veterinary Research Communication, Dordrecht, v. 31, n. 1, p. 53-65, 2007.

LINDGREN, K. et al. Parascaris equorum in foals and in their environment on a Swedish stud farm, with notes on treatment failure of ivermectin. Veterinary Parasitology, Amsterdam, v. 151, n. 2/ 4, p. 337-343, 2008.

MAT'THEWS, J. B. et al. Recent developments in research into the cyathostominae and Anoplocephala perfoliata. Available: <www.vetres.org/index.php?option=article\&access=standard\&Itemid=129\&url=/ articles/vetres/pdf/2004/04/V4404.pdf>. Access: 30 June 2007. 
NIELSEN, M. K. et al. Detection and semi-quantification of Strongylus vulgaris DNA in equine faeces by real-time quantitative PCR. International Journal for Parasitology, New York, v. 38, n. 3/4, p. 443-453, 2008.

PEREGRINE, A. S. et al. Larval ctathostominosis in horses in Ontario: An emerging disease? Canadian Veterinary Journal, Ottawa, v. 46, n. 1, p. 80-82, 2005.

PEREIRA, J. R.; VIANNA, S. S. S. Gastrointestinal parasitic worms in equines in the Paraíba Valley, State of São Paulo, Brazil. Veterinary Parasitology, Amsterdam, v. 140, n. 3/4, p. 289$295,2006$.

RIET-CORREIA, F. et al. Doenças de ruminantes e equinos. 2. ed. São Paulo: Varela, 2001. v. 2, p. 32-146.

SAMSON-HIMMELSTJERNA, G. von. et al. Cases of reduced cyathostomin egg-reappearance period and failure of Parascaris equorum egg count reduction following ivermectin treatment as well as survey on pyrantel efficacy on German horse farms. Veterinary Parasitology, Amsterdam, v. 144, n. 1/2, p. 74-80, 2007.

SLOCOMBE, J. O. D.; GANNES, R. V. G. de; LAKE, M. C. Macrocyclic lactone-resistant Parascaris equorum on stud farms in Canada and effectiveness of fenbendazole and pyrantel palmoate. Veterinary Parasitology, Amsterdam, v. 145, n. 3/4, p. 371-376, 2007.

SMITH, B. P. Tratado de medicina interna de grandes animais. São Paulo: Manole, 1994.

URQUHART, G. M. Parasitologia veterinária. 2. ed. Rio de Janeiro: Guanabara Koogan, 1998.

XUAN, X. et al. Diagnosis of equine piroplasmosis in Brazil by serodiagnostic methods with recombinant antigens. Journal of Veterinary Medical Science, Tokyo, v. 63, n. 10, p. 1159-1160, 2001.

Recebido: 01/03/2009

Received: 03/01/2009

Aprovado: 25/04/2009

Approved: 04/25/2009 